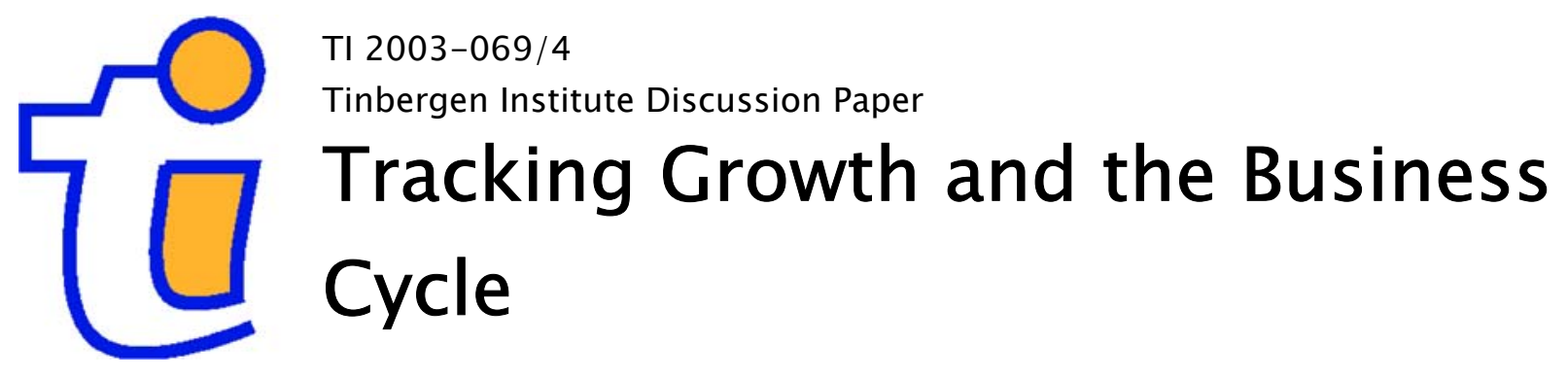

João Valle e Azevedo'

Siem Jan Koopman',2

António Rua ${ }^{3}$

\footnotetext{
' Department of Econometrics, Free University, Amsterdam,

2 Tinbergen Institute,

${ }^{3}$ Economic Research Department, Banco de Portugal, Lisboa.
} 


\section{Tinbergen Institute}

The Tinbergen Institute is the institute for economic research of the Erasmus Universiteit Rotterdam, Universiteit van Amsterdam, and Vrije Universiteit Amsterdam.

Tinbergen Institute Amsterdam

Roetersstraat 31

1018 WB Amsterdam

The Netherlands

Tel.: $\quad+31(0) 205513500$

Fax: $\quad+31(0) 205513555$

Tinbergen Institute Rotterdam

Burg. Oudlaan 50

3062 PA Rotterdam

The Netherlands

Tel.: $\quad+31(0) 104088900$

Fax: $\quad+31(0) 104089031$

Please send questions and/or remarks of nonscientific nature to driessen@tinbergen.nl.

Most TI discussion papers can be downloaded at http://www.tinbergen.nl. 


\title{
Tracking growth and the business cycle: a stochastic common cycle model for the Euro area
}

João Valle e Azevedo ${ }^{(a)}$, Siem Jan Koopman ${ }^{(a),(b) *}$ and António Rua ${ }^{(c)}$

${ }^{(a)}$ Department of Econometrics, Free University, Amsterdam

(b) Tinbergen Institute, Amsterdam

(c) Economic Research Department, Banco de Portugal, Lisboa

August 27, 2003

\begin{abstract}
This paper proposes a new model-based method to obtain a coincident indicator for the business cycle. A dynamic factor model with trend components and a common cycle component is considered which can be estimated using standard maximum likelihood methods. The multivariate unobserved components model includes a stationary higher order cycle. Also higher order trends can be part of the analysis. These generalisations lead to a business cycle that is similar to a band-pass one. Furthermore, cycle shifts for individual time series are incorporated within the model and estimated simultaneously with the remaining parameters. This feature permits the use of leading, coincident and lagging variables to obtain the business cycle coincident indicator without prior analysis of their lead-lag relationship. Besides the business cycle indicator, the model-based approach also allows to get a growth rate indicator. In the empirical analysis for the Euro area, both indicators are obtained based on nine key economic time series including gross domestic product, industrial production, unemployment, confidence indicators and interest rate spread. This analysis contrasts sharply with earlier multivariate approaches. In particular, our more parsimonious approach leads to a growth rate indicator for the Euro area that is similar to the one of EuroCOIN. The latter is based on a more involved approach by any standard and uses hundreds of time series from individual countries belonging to the Euro area.
\end{abstract}

Keywords: Band-pass filter; Coincident indicator; Dynamic factor model; Kalman filter; Leading indicator; Unobserved components time series model; Phase shift; Revisions. JEL classification: C13, C32, E32.

*Corresponding author: Department of Econometrics, Free University Amsterdam, De Boelelaan 1105, 1081 HV Amsterdam, The Netherlands. Emails: azevedoj@stanford.edu s.j.koopman@feweb.vu.nl antonio.rua@bportugal.pt. 


\section{Introduction}

Undertaking fiscal and monetary policies requires information about the state of the economy. Given the importance of this knowledge for the policymaker, a clear economic picture needs to be present at any time. However, the assessment of the economic situation can be a challenging task when one faces noisy data giving mixed signals about the overall state of the economy. In order to avoid a solely judgemental based procedure in the economic analysis one should be able to extract the relevant information through a statistical rigorous method capable of providing a clear signal regarding current and future economic developments. Moreover, since data is often not available at the same and/or desired frequency, one has also to be able to reconcile a high frequency business cycle indicator without disregarding data recorded at a lower frequency. The crucial example refers to gross domestic product (GDP). Real GDP is undoubtedly one of the most important variables for business cycle assessments but it is usually discarded in the construction of composite indicators presumably because it is available only on a quarterly basis (see, for example, Stock and Watson (1989)). Others, such as Altissimo, Bassanetti, Cristadoro, Forni, Lippi, Reichlin, and Veronese (2001), overcome this shortcoming by using a GDP monthly series obtained through the linear interpolation of the quarterly figures. In order to avoid such an ad-hoc procedure we will treat quarterly series as monthly series with missing observations. It allows the construction of a business cycle indicator that is based on both quarterly and monthly series without prior transformation of the data. A similar approach for the construction of the coincident index of Stock and Watson (1989) is proposed by Mariano and Murasawa (2003).

In this paper we propose a new method for obtaining a business cycle indicator. It is a modelbased approach and the model falls within the class of multivariate unobserved components time series models which can be expressed in state-space form and estimated by maximum likelihood via the Kalman filter; see, for example, Harvey (1989) and Durbin and Koopman (2001). The model enables the decomposition of the time series into trend, cycle and irregular components. Recently, Harvey and Trimbur (2003) have presented a new class of model-based filters for

extracting trends and cycles in economic time series. The main feature of these model-based filters is that it is possible to extract a smooth cycle from economic time series, likewise a band-pass filter. They have shown that the implied filters from such models include, as special cases, some of the filters used in business cycle analysis (such as the Hodrick and Prescott (1997) filter) and since they are model-based they provide a mutually consistent way to extract trends and cycles even at the beginning and end of the series. In view of this, they overcome one of the major drawbacks of the well-known Baxter and King (1999) band-pass filter for realtime macroeconomic analysis. Although recently Christiano and Fitzgerald (2003) provide a solution to the endpoints problem, such an univariate approach does not exploit the information stemming from several economic time series, which can be available at different frequencies.

Apart from modelling trends and cycles of individual time series in a multivariate context, interest does also focus on modelling lead-lag relationships between variables and the business 
cycle. For this purpose the multivariate cycle model is generalised to allow individual cycles to be shifted with respect to a base cycle, see Rünstler (2002). This generalisation enables the simultaneous modelling of leading, coincident and lagging indicators for the business cycle without their a priori classification, i.e. the phase shifts are estimated as parameters of the model. The resulting common factor for the cycle is the business cycle indicator. This contrasts with previous literature on multivariate unobserved components time series models where the coincident indicator is defined as the common factor of a small set of coincident variables identified prior to estimation (see, for example, Stock and Watson (1989)). The simultaneous treatment of phase shifts in a model-based approach allows to exploit the information contained in both lagging and leading variables to obtain the business cycle coincident indicator.

This novel approach to business cycle and growth tracking is applied to Euro area economic data. By pooling information from nine key economic variables (including GDP, industrial production, retail sales, confidence indicators, among others) it is possible to obtain a business cycle indicator in line with common wisdom regarding Euro area business cycle developments. Besides the business cycle indicator, which can be seen as a monthly proxy for the output gap, one can obtain as a by-product the corresponding growth rate indicator. The resulting growth rate indicator can be compared with EuroCOIN, which is a coincident indicator for the Euro area economic activity suggested by Altissimo, Bassanetti, Cristadoro, Forni, Lippi, Reichlin, and Veronese (2001). EuroCOIN is based on a generalised dynamic factor model resorting to frequency domain analysis to account for phase shifts between the variables and uses as input almost a thousand series. We find that our more parsimonious approach leads to similar results.

The remaining of the paper is organised as follows. In section 2 we present the underlying unobserved components basic model and the corresponding extensions. In section 3 we proceed to the empirical application for the Euro area. Section 4 concludes.

\section{The stochastic common cycle model}

\subsection{Basic model}

Assume that a panel of $N$ economic time series are collected in the $N \times 1$ vector $y_{t}$ and that we observe $n$ data points over time, that is $t=1, \ldots, n$. The $i$-th element of the observation vector at time $t$ is denoted by $y_{i t}$.

The basic unobserved components time series model for measuring the business cycle from a panel of economic time series can be based on the specification

$$
y_{i t}=\mu_{i t}+\delta_{i} \psi_{t}+\varepsilon_{i t}, \quad i=1, \ldots, N, \quad t=1, \ldots, n,
$$

where $\mu_{i t}$ is the individual trend component for the $i$ th time series and the business cycle component (common to all time series) is denoted by $\psi_{t}$. For each series the contribution of the cycle is measured by the coefficient $\delta_{i}$ for $i=1, \ldots, N$. The idiosyncratic disturbance $\varepsilon_{i t}$ is assumed normal and independent from $\varepsilon_{j s}$ for $i \neq j$ and/or $t \neq s$. The variance of the irregular 
disturbance varies for the different individual series, that is

$$
\varepsilon_{i t} \sim \mathcal{N} \mathcal{I} \mathcal{D}\left(0, \sigma_{i, \varepsilon}^{2}\right), \quad i=1, \ldots, N
$$

Each trend component from the panel time series is specified as the local linear trend component (see Harvey, 1989), that is

$$
\begin{aligned}
& \mu_{i, t+1}=\mu_{i t}+\beta_{i t}+\eta_{i t}, \quad \eta_{i t} \sim \mathcal{N} \mathcal{I D}\left(0, \sigma_{i, \eta}^{2}\right), \\
& \beta_{i, t+1}=\beta_{i t}+\zeta_{i t}, \quad \zeta_{i t} \sim \mathcal{N} \mathcal{I D}\left(0, \sigma_{i, \zeta}^{2}\right),
\end{aligned}
$$

for $i=1, \ldots, N$. The disturbances $\eta_{i t}$ and $\zeta_{i t}$ are serially and mutually independent of each other, and other disturbances, at all time points and across the $N$ equations. The trends are therefore independent of each other within the panel. A smooth trend specification is obtained by setting $\sigma_{i, \eta}^{2}=0$ so that the trend component reduces to an integrated random walk process. Since our focus is on the business cycle we do not attempt to find common dynamics in the trend components. However, it can be done in this setting of multivariate models; see Harvey and Koopman (1997). The dynamic (nonstationary) properties of a single local linear trend model are well established and explored in Harvey (1989) and used in empirical work, for example, by Harvey and Jaeger (1993).

The cycle component is common to all time series in the panel and can be specified as an autoregressive model with polynomial coefficients that have complex roots. Specifically, we enforce this restriction by formulating the cycle time series process as a trigonometric process, that is

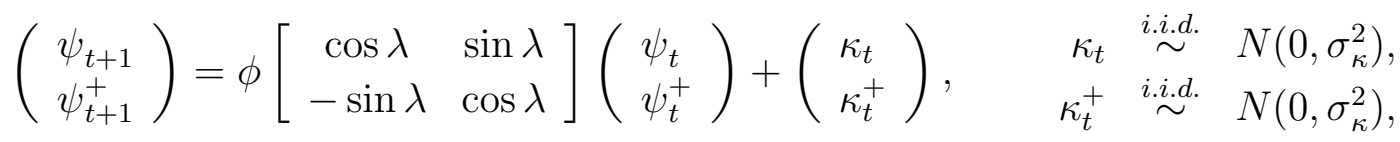

where $\kappa_{t}$ and $\kappa_{t}^{+}$are white noise disturbances and mutually independent of each other at all time points for $t=1, \ldots, n$. The damping term $0<\phi \leq 1$ ensures that the stochastic process $\psi_{t}$ is stationary or fixed as the cycle variance is specified as $\sigma_{\kappa}^{2}=\left(1-\phi^{2}\right) \sigma_{\psi}^{2}$ where $\sigma_{\psi}^{2}$ is the variance of the cycle. The overall frequency of the stochastic cycle is $0 \leq \lambda \leq \pi$ which implies that the period of the cycle is $2 \pi / \lambda$. The initial distribution of the cycle is given by

$$
\psi_{1} \stackrel{i . i . d .}{\sim} N\left(0, \sigma_{\psi}^{2}\right), \quad \psi_{1}^{+} \stackrel{i . i . d .}{\sim} N\left(0, \sigma_{\psi}^{2}\right)
$$

where $\sigma_{\psi}^{2}=\sigma_{\kappa}^{2} /\left(1-\phi^{2}\right)$. The dynamic properties of the cycle can be characteristed via the autocovariance function which is given by

$$
\gamma(\tau)=E\left(\psi_{t} \psi_{t-\tau}\right)=\sigma_{\kappa}^{2} \phi^{\tau}\left(1-\phi^{2}\right)^{-1} \cos (\lambda \tau)
$$

for $\tau=0,1,2, \ldots$.

The model falls within the class of multivariate unobserved components time series models (or multivariate structural time series models) and can be casted in state space form. Estimation 
of parameters (including variances associated with the disturbances of the model and loading factor coefficients) and estimation of unobservables such as trends and cycles can be based on Kalman filter and associated smoothing methods. An introduction to such techniques is found in, for example, Harvey (1989) and Durbin and Koopman (2001).

The basic structure of the model is simple but not adequate to capture the business cycle for the following reasons. First, the individual cycles of the economic series in the panel will be correlated with each other but not necessarily contemporaneously. Lead and lag relationships may be more adequate as they are often found in empirical work. Model (1) does not account for lead and lagged relationships between cycles. Stronger relations between cycles can be obtained when cycles are leaded or lagged (that is, shifted forwards or backwards). To allow for shifted cycles we follow a recent contribution of Rünstler (2002) that implements a modification of the multivariate cycle component. It allows individual cycles to be shifted with respect to a base cycle. In the next section we will discuss the details of this generalisation. Second, the model may have difficulty in capturing the business cycle dynamics given the dominant role played by low frequencies in macroeconomic time series. These frequencies may mask business cycle dynamics. However, recently developed specifications for the trend and cycle can result in an estimated cycle with properties that are closely associated with a band-pass filtered cycle; see Gomez (2001) and Harvey and Trimbur (2003). In this paper we adopt the new formulations in a multivariate setting and we generalise the multivariate common cycle model by introducing shifts.

\subsection{Shifted cycles}

By considering the cycle process (4) and by using a standard trigonometric identity, it can be shown that a cycle is shifted $\xi_{0}$ time periods to the right (when $\xi_{0}>0$ ) or to the left (when $\left.\xi_{0}<0\right)$ by computing

$$
\cos \left(\xi_{0} \lambda\right) \psi_{t}+\sin \left(\xi_{0} \lambda\right) \psi_{t}^{*}
$$

where $-\frac{1}{2} \pi<\xi_{0} \lambda<\frac{1}{2} \pi$. The shift is with respect to $\psi_{t}$ and the restrictive parameter space for $\xi_{0}$ follows from the periodic nature of trigonometric functions. The cycle shift operation is introduced by Rünstler (2002) for the multivariate structural time series model with a stochastic similar cycle as introduced by Harvey and Koopman (1997). It is further shown that the shift operation is also valid for a common cycle such as the one present in model (1). Shifted cycles are introduced in the model via the formulation

$$
y_{i t}=\mu_{i t}+\delta_{i}\left\{\cos \left(\xi_{i} \lambda\right) \psi_{t}+\sin \left(\xi_{i} \lambda\right) \psi_{t}^{+}\right\}+\varepsilon_{i t}, \quad i=1, \ldots, N, \quad t=1, \ldots, n,
$$

where the specifications for $\mu_{i t}, \psi_{t}$ (with associated variable $\psi_{t}^{+}$) and $\varepsilon_{i t}$ remain unaltered as in (3), (4) and (2), respectively. The shifts are modelled as shifts in the cross autocorrelation function for different cycles. In case of the shifted common cycle component, the cross autocovariance is given by

$$
\begin{aligned}
\gamma_{i j}(\tau) & =E\left(\psi_{i t} \psi_{j, t-\tau}\right) \\
& =\sigma_{\kappa}^{2} \phi^{\tau}\left(1-\phi^{2}\right)^{-1} \delta_{i} \delta_{j} \cos \left\{\lambda\left(\tau+\xi_{i}-\xi_{j}\right)\right\}, \quad i, j=1, \ldots, N,
\end{aligned}
$$


for $\tau=0,1, \ldots$ and where

$$
\psi_{i t}=\delta_{i}\left\{\cos \left(\xi_{i} \lambda\right) \psi_{t}+\sin \left(\xi_{i} \lambda\right) \psi_{t}^{+}\right\}
$$

This result is a direct consequence of the general results given in Rünstler (2002, Proposition 2).

The contemporaneous cross-correlation between two cycles is $\gamma_{i j}(0) / \sqrt{\gamma_{i i}(0) \gamma_{j j}(0)}=\cos \left\{\lambda\left(\xi_{i}-\right.\right.$ $\left.\left.\xi_{j}\right)\right\}$. However, when cycles are shifted accordingly, the correlation is $\cos (0)=1$ as expected from a common cycle specification. It is important to realise that a real-time shift $\xi_{i}$ is rarely estimated as an integer time point and since observed time series data can only be shifted at discrete time intervals the contemporaneous cross-correlation of cycles associated with shifted time series will usually not be one.

The individual cycles can only be shifted with respect to another cycle. Therefore the first equation is subject to parameter restrictions $\xi_{1}=0$ and $\delta_{1}=1$ and given by

$$
y_{1 t}=\mu_{1 t}+\psi_{t}+\varepsilon_{1 t}, \quad t=1, \ldots, n
$$

The shifts and loads of the other equations are all relative to the cycle of the first equation. It follows that this cycle is common to the other time series but scaled differently and shifted by $\xi_{i}$ time points. Of course, the parameter restrictions can also be applied to another equation. Finally, the shift parameter $\xi_{i}(i=2, \ldots, N)$ is identified and can be estimated by standard maximum likelihood since the joint covariance structure of $y_{1}, \ldots, y_{n}$ is affected by $\xi_{i}$, which follows from (7), provided that $\delta_{i} \neq 0$ for equations $i=1, \ldots, N$.

\subsection{Generalised components for model-based band-pass filtering}

The basic trend plus cycle decomposition model (1) for time series $i$ can be generalised such that the model becomes the basis for extracting trends and cycles with band-pass filtering properties. For example, the $m$-th order stochastic trend for series $i$ can be considered which is given by $\mu_{i t}=\mu_{i t}^{(m)}$ where

$$
\Delta^{m} \mu_{i, t+1}^{(m)}=\zeta_{i t}, \quad \zeta_{i t} \sim \mathcal{N} \mathcal{I D}\left(0, \sigma_{i, \zeta}^{2}\right)
$$

or

$$
\mu_{i, t+1}^{(j)}=\mu_{i t}^{(j)}+\mu_{i t}^{(j-1)}, \quad j=m, m-1, \ldots, 1,
$$

with $\mu_{i t}^{(0)}=\zeta_{i t}$ as given by (8) for $i=1, \ldots, N$ and $t=1, \ldots, n$. The trend specifation (8) can be taken as the model representation of the class of Butterworth filters that are commonly used in engineering, see Gomez (2001). The special case of (8) with $m=2$ reduces to the local linear trend component (3) with $\sigma_{i, \eta}^{2}=0$ from which it follows that $\beta_{i t}=\mu_{i t}^{(1)}$. This specification is sometimes referred to as the smooth linear trend or the integrated random walk, see Young (1984) and Kitagawa and Gersch (1996). The effect of a higher value for $m$ is pronounced in the frequency domain since the low-pass gain function will have a sharper cut-off downwards at a certain low frequency point. In the time domain the effect is evident since $\Delta^{m} \mu_{i, t+1}^{(m)}=\zeta_{i t}$ 
suggests that the time series $y_{i t}$ implied by model (1) with (8) needs to be differenced $m$ times to become stationary. From this perspective, the choice of a relatively large $m$ may therefore not be realistic for most economic time series.

The generalised specification for the $k$-th order common cycle component is given by $\psi_{t}=$ $\psi_{t}^{(k)}$ where

$$
\left(\begin{array}{c}
\psi_{t+1}^{(j)} \\
\psi_{t+1}^{+(j)}
\end{array}\right)=\phi\left[\begin{array}{cc}
\cos \lambda & \sin \lambda \\
-\sin \lambda & \cos \lambda
\end{array}\right]\left(\begin{array}{l}
\psi_{t}^{(j)} \\
\psi_{t}^{+(j)}
\end{array}\right)+\left(\begin{array}{c}
\psi_{t}^{(j-1)} \\
\psi_{t}^{+(j-1)}
\end{array}\right), \quad j=1, \ldots, k,
$$

with

$$
\psi_{t}^{0}=\kappa_{t} \stackrel{i . i . d .}{\sim} N\left(0, \sigma_{\kappa}^{2}\right), \quad \psi_{t}^{+0}=\kappa_{t}^{+i . i . d .} N\left(0, \sigma_{\kappa}^{2}\right),
$$

for $t=1, \ldots, n$. Different variations within this class of generalised cycles are discussed by Harvey and Trimbur (2003) who refer to specification (10) as the balanced cyclical model. They prefer the balanced specification since the time-domain properties of cycle $\psi_{t}$ are more straightforward to derive and since it tends to give a slightly better fit to a selection of U.S. economic time series. Given the choice of the order of the cycle $k$, the number of unknown parameters remain three as in (4), that is $0<\phi \leq 1,0 \leq \lambda \leq \pi$ and $\sigma_{\kappa}^{2}>0$. The initial condition of the cycle component, that is the unconditional distribution of the cycle process (10), can be obtained analytically, with details provided by Trimbur (2002), or numerically; see Appendix A for more details. In the same paper the autocovariance function of the generalised cycle is reported as

$$
\gamma(\tau)=\sigma_{\kappa}^{2} \phi^{\tau} \cos (\lambda \tau) g(\phi, \tau)
$$

where

$$
g(\phi, \tau)=\sum_{i=k-\tau}^{\tau}\left(1-\phi^{2}\right)^{1-i-k}\left(\begin{array}{c}
\tau \\
k-i
\end{array}\right) \sum_{j=0}^{i-1} \phi^{2 j}\left(\begin{array}{c}
i-1 \\
j
\end{array}\right)\left(\begin{array}{c}
k-1 \\
j+k-i
\end{array}\right),
$$

for a $k$-th order cycle and for $\tau=0,1,2, \ldots$; see Trimbur (2002, Proof of proposition 2).

In the case of two correlated cycles (whether correlation is perfect or not), it follows from below that the shift mechanism of Rünstler (2002) can also be applied to the generalised cycle specification. This means that cycles with band-pass filter properties can be subject to shifts within a multivariate cycle model based on the generalised cycle component (10). In the case of model (6), the properties of the cycle component for the first series in the panel will be closely related to those of the univariate band-pass filtered series. However, the information from the other series is also exploited in the estimation of the cycle component. Finally, the cross autocovariance function of the generalised shifted cycle component is given by

$$
\gamma_{i j}(\tau)=\sigma_{\kappa}^{2} \phi^{\tau} \delta_{i} \delta_{j} \cos \left\{\lambda\left(\tau+\xi_{i}-\xi_{j}\right)\right\} g(\phi, \tau)
$$

for $\tau=0,1,2, \ldots$. This result follows straightforwardly by applying Proposition 2 of Rünstler (2002) to the proof of Proposition 2 of Trimbur (2002). Since the autocovariance function for generalised cycles also applies to negative discrete values for $\tau$ and it is symmetric in $\tau=0$, the shift mechanism has the desired effect on the autocovariance function of the shifted cycle; see Rünstler (2002, Proof of Proposition 2). 


\section{Empirical results for the Euro Area}

\subsection{Definition of business cycle and data}

The multivariate framework presented in the previous section allows the simultaneous modelling of leading, coincident and lagging indicators for the business cycle with phase shifts being estimated as an integrated part of the modelling process. However, as a preliminary step, one has to define the base cycle. We follow Stock and Watson (1999) who consider that "... fluctuations in aggregate output are at the core of the business cycle so the cyclical component of real GDP is a useful proxy for the overall business cycle ...". Therefore, we impose a unit common cycle loading and a zero phase shift for Euro area real GDP. The remaining series included in the multivariate analysis are the most commonly used monthly indicators on Euro area economic monitoring. This includes industrial production, retail sales, unemployment, several confidence indicators (consumers, industrial, retail trade and construction) and interest rate spread ${ }^{1}$. As it is well known, industrial production is highly correlated with the business cycle and it is coincident or even slightly leading. On the other hand, retail sales provide information about private consumption, which accounts for a large proportion of GDP. Unemployment is a countercyclical and lagging variable but it also displays a high correlation with the business cycle. Confidence indicators provide information regarding economic agents' assessment of current and future economic situation so they are expected to present coincident or leading properties. Finally, the interest rate spread has been widely recognised as a forward-looking indicator for real activity. These time series are available for the period between 1986 and 2002 and graphically presented in figure 1 . The resulting dataset covers different economic sectors and it is based on both quantitative and qualitative data as well as on both real and monetary variables. As pointed out earlier, GDP is available only on quarterly basis whereas the other indicators are monthly. However, as it is well known, one can deal with the missing observations problem easily in the state space framework (see, for example, Harvey (1989)). Thus, this model-based approach offers the advantage of avoiding the use of an ad-hoc interpolation procedure to get a monthly GDP series prior to multivariate analysis.

\subsection{Details of model and estimation}

The empirical business cycle analysis for the Euro area will be based on model (6) with generalised trend $\mu_{i t}$ and common cycle $\psi_{t}$ given by model specifications (8) and (10), respectively. The first series in the panel is real GDP. The time index refers to months although specific series (such as GDP) may be measured only every quarter. Since the model will be cast in state space for estimation and signal extraction, the treatment of variables with different observation frequencies in the panel can be handled straightforwardly using the Kalman filter. The treatment of quarterly time series amounts to having two consecutive monthly observations as missing followed by the actual quarterly observation. We do not allow for the accounting identity of

\footnotetext{
${ }^{1}$ Appendix B provides a detailed description of the data.
} 


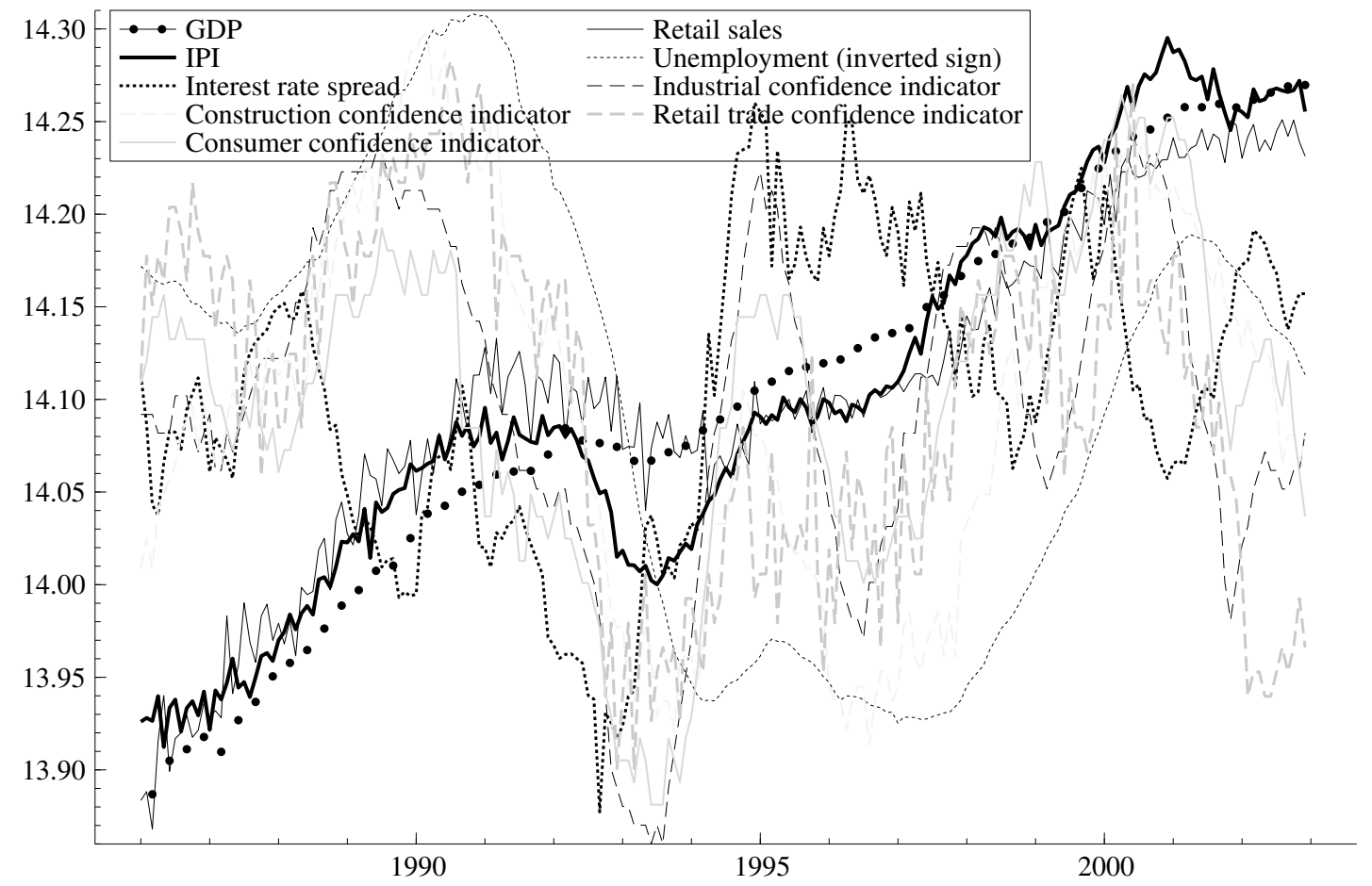

Figure 1: Nine key economic variables for the Euro area between 1986 and 2002.

three months in one quarter as in Mariano and Murasawa (2003) since GDP is measured in logs and we are merely interested in the cycle and growth dynamics of GDP.

We have set $m=2$ and $k=6$ since Harvey and Trimbur (2003) show that this choice leads to estimated trend and cycle components with band-pass filtering properties similar to the ones of Baxter and King (1999). The role of the trend component $\mu_{i t}$ in model (6) is to capture the persistence of the time series excluding the cycle. The order $m$ of the trend (8) applies to all time series in the panel. The frequency of the common cycle component is fixed to assure that the cycle captures the typical business cycle period in the time series. The frequency is therefore chosen as $\lambda=0.06545$ which implies a period of 96 months ( 8 years), see Stock and Watson (1999) for the U.S. and the ECB (2001) for the Euro area. The shift $\xi_{i}$ is measured in real-time so that $\xi_{i} \lambda$ is measured in radians and due to the periodicity of trigonometric functions the parameter space of $\xi_{i}$ is within the range $-\frac{1}{2} \pi<\xi_{i} \lambda<\frac{1}{2} \pi$ for $i=2, \ldots, N$. For example, it follows that when a cosine is shifted by $x \lambda$, its correlation with the base cosine is the same as its correlation with a cosine that is shifted by $(\pi-x) \lambda$ but with an opposite sign, for $\frac{1}{2} \pi<x \lambda<\pi$. Further, we note that $\delta_{1}$ and $\xi_{1}$, the load and shift parameters associated with the base series GDP, are restricted to one and zero, respectively. Apart from these restrictions, the number of parameters to estimate for each equation is four $\left(\sigma_{i, \zeta}^{2}, \delta_{i}, \xi_{i}\right.$ and $\left.\sigma_{i, \varepsilon}^{2}\right)$ and for the common cycle is two $\left(\phi\right.$ and $\sigma_{\kappa}^{2}$ ). The total number therefore is $4 N$ ( 4 for each equation minus two restrictions plus two for the common cycle). 
The model specification can be casted in state space for which the details are given in Appendix A. The maximum likelihood estimates of the parameters are obtained by maximising the loglikelihood function that is evaluated through the Kalman filter. This is a standard exercise although computational complexities may arise because the parameter dimension rises quickly as $N$ increases. For example, with $N=9$ the number of paramaters to be estimated is 36. In principle, there is no difficulty of being able to empirically identify the parameters from the data since the number of observations increases accordingly with $N$. It can however be computationally and numerically cumbersome to obtain the maximum value of the loglikelihood function in a 36 dimensional space ${ }^{2}$.

Once the model parameters are estimated, the trends and the common business cycle can be extracted from the data using the Kalman filter and associating smoothing algorithms. The Kalman filter is sometimes known to be slow for multivariate models, especially when specific modifications are considered for the diffuse initialisations of nonstationary components such as trends. However, we would like to stress that the implementation of the Kalman filter for multivariate models as documented in Koopman and Durbin (2000) and implemented in SsfPack ${ }^{3}$ was not slow nor numerically unstable.

\subsection{The business cycle coincident indicator}

The estimated components for Euro area real GDP are presented in figure 2. Several interesting features become apparent. First, although GDP is recorded on a quarterly basis the estimated components are monthly. These components can be seen as the outcome of the underlying monthly GDP decomposition which can be recovered resorting to the information contained in the remaining dataset. Second, by analysing the trend it seems that Euro area potential growth has declined after the major recession that occurred in 1993. This feature becomes clear by plotting the trend slope against the time axis. One can see that before the recession monthly potential growth was around 0.3 per cent (3.7 per cent in annualised terms) while afterwards it was closer to 0.2 per cent (2.4 per cent in annualised terms, which falls within the $2.0-2.5$ per cent GDP potential growth range underlying the ECB monetary policy). Third, the resulting GDP cyclical component, i.e. our Euro area business cycle indicator, seems to be in line with the common wisdom regarding Euro area business cycle developments (see ECB (2002)). In particular, a major recession was recorded in 1993 (as German unification boost

\footnotetext{
${ }^{2} \mathrm{~A}$ practical and feasible approach to overcome some numerical difficulties is to obtain starting values from univariate and bivariate models. Consecutively, the model dimension can be increased step by step and for each step realistic starting values will be available from the earlier low-dimensional models. This approach can be fully automated and different orderings in the panel of time series can be taken in order to be assured (at least, to some extent) that the maximum is obtained. Further, the maximisation process is stopped at specific parameter values and restarted at different and realistic values in order to safeguard the estimation process from producing unrealistic model estimates.

${ }^{3}$ All computations for this paper are done using the object-oriented matrix language Ox of Doornik (2001) together with the state space functions in version 3 of SsfPack as developed by Koopman, Shephard, and Doornik (1999) (for later versions, see http://www.ssfpack.com).
} 
delayed the impact of the recessions in the United States and the United Kingdom in the early 1990s) while there was an economic slowdown in the late 1990s following the crisis in Asian emerging market economies and the financial instability in Russia. Although the aim of the suggested model-based approach is to obtain a coincident indicator for the business cycle, one can also get a leading one. By repeatedly applying the Kalman filter prediction equations it is possible to obtain multi-step prediction equations. Thus, one can define the leading indicator as the $h$-step ahead forecast of the coincident indicator with $h$ chosen as desired but typically not implying a lead of more than half year (see, for example, Stock and Watson (1989)).
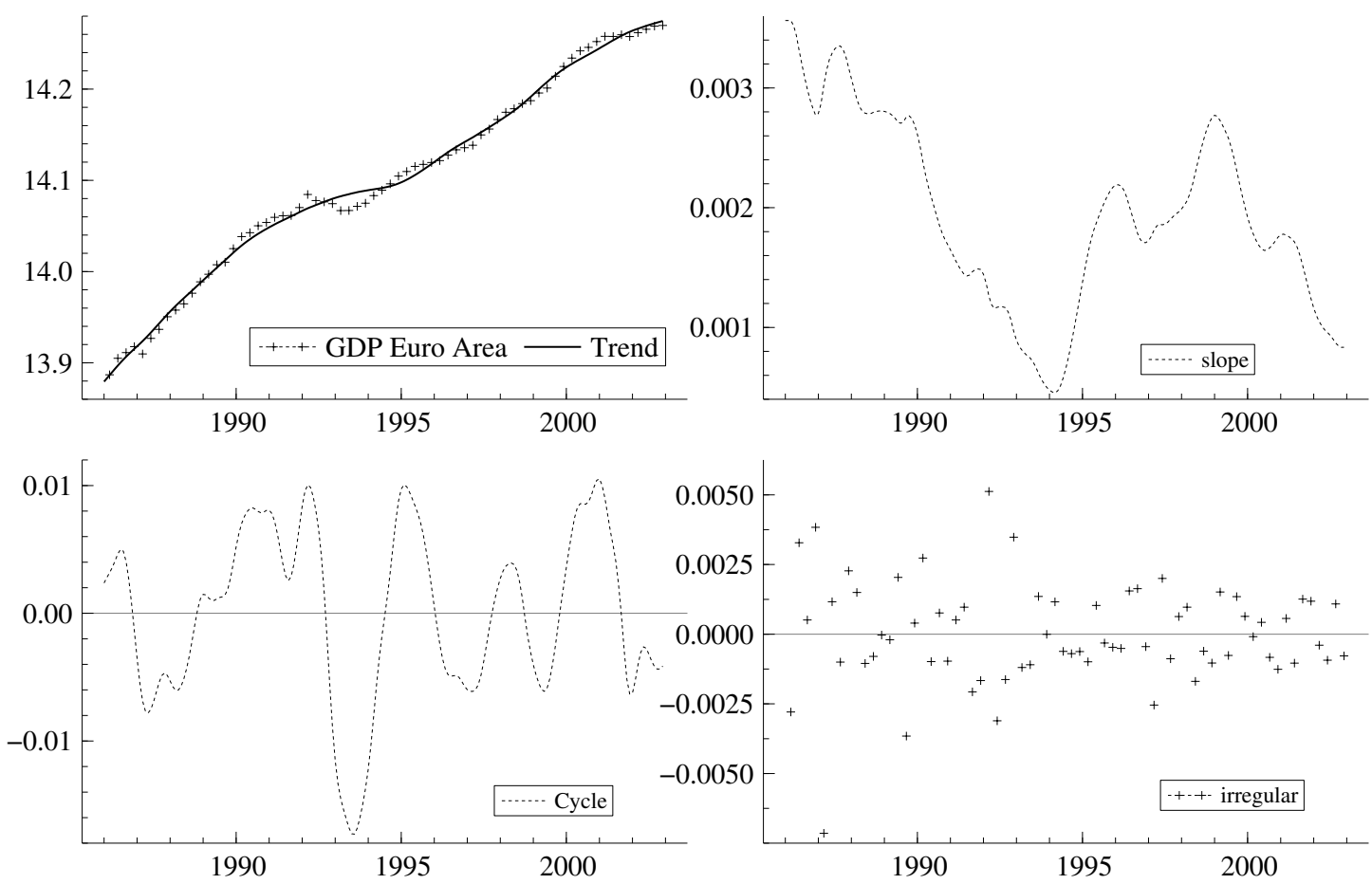

Figure 2: Decomposition of real GDP: GDP and trend, trend slope, cycle and irregular.

The variances of the trends and the damping factor and variance of the common cycle are estimated. For the latter parameters we obtained estimates $\hat{\sigma}_{\omega}=0.0066$ and $\hat{\phi}=0.57$ which are typical values for a generalised cycle with $m=6$, see the discussion in Harvey and Trimbur (2003). The resulting properties of the estimated cycle are therefore similar to bandpass filtered cycles such as the ones of Baxter and King (1999) and Christiano and Fitzgerald (2003). The variances of the individual trends are also estimated and they vary from very small values (suggesting that trend is fixed and may coincide with the fact that trend does not exist in the series) to relatively high values (suggesting that trend and its corresponding slope change throughout the series). Some key estimation results are presented in table 1 . The load factors are of interest since they indicate the relative contribution of the series to the construction of the cycle component. Since the series have different scales of measurement, the 
reported loads are standardised by the sample variance of the corresponding differenced series. It is interesting to observe that industrial confidence tops the list of contributors (others than GDP itself) to the construction of the estimated business cycle while industrial production, consumer confidence and construction confidence are important too. Series related to retail trade contribute relatively little to the estimated business cycle.

The ability to simultaneously identify and estimate cycle shifts within the model for a given set of time series is a key development that is important for the current analysis. The estimation results reveal that unemployment lags the business cycle by more than one year (almost 16 months) while interest rate spread clearly leads the business cycle by almost 17 months. The common cycle component for the other series displays relatively small shifts, confirming the coincident character of these series.

In order to provide some indication whether the multivariate trend-cycle model fits the individual series successfully, we report goodness-of-fit statistics in table 1. The sum of squared prediction errors is compared to the sample variance of the differenced time series for each equation of the model. The reported statistics give evidence that in fact all time series (to different degrees) are appropriately described by our model.

Table 1: Selected estimation results

\begin{tabular}{lccc}
\hline time series & stand. load & shift & goodness of fit \\
\hline \hline Gross domestic product & - & - & 0.31 \\
Industrial production & 1.18 & 6.85 & 0.67 \\
Unemployment & -0.42 & -15.9 & 0.78 \\
Industrial confidence & 2.46 & 7.84 & 0.47 \\
Construction confidence & 0.77 & 1.86 & 0.51 \\
Retail trade confidence & 0.26 & -0.22 & 0.67 \\
Consumer confidence & 1.12 & 3.76 & 0.33 \\
Retail sales & 0.11 & -4.70 & 0.86 \\
Interest rate spread & 0.57 & 16.8 & 0.22 \\
\hline
\end{tabular}

The stand. load is the maximum likelihood estimate of coefficient $\delta_{i}$ divided by the standard deviation of the differenced time series $i$. Shift is the maximum likelihood estimated of shift parameter $\xi_{i}$. Note that for gross domestic product load $\delta_{1}$ is fixed at one and shift $\xi_{1}$ is fixed at zero. Goodness of fit is the coefficient of determination $\left(R^{2}\right)$ computed as one minus the ratio of the variance of the one-step ahead prediction residuals for equation $i$ (sample mean is substracted) and the sample variance of the differenced time series $i$.

One of the main focus of the paper is on extracting the business cycle using multiple economic time series. Therefore, we present the business cycle coincident indicator once more in figure 3 together with the Euro area business cycle turning points settled by the European Central Bank, see ECB (2001). However, since the ECB dating was made on a quarterly basis, the quarters where presumably occured a turning point are represented by a shaded area due to 
the monthly nature of the time axis. One can see that the suggested business cycle coincident indicator tracks, in general, quite well the turning points. Only at the end of the sample it seems to perform less satisfactorily. However, one should note that the ECB dating was made using data only up to 2000. In particular, this can explain why the last peak dated by ECB is not confirmed by our business cycle indicator since it suggests that the peak only occurred at the beginning of 2001.

Some additional descriptive statistics of the extracted business cycle are reported in table 2. The historical minimum value of the business cycle is observed in August 1993, which falls within the most severe recession period recorded in the Euro area, while the maximum value is at January 2001.

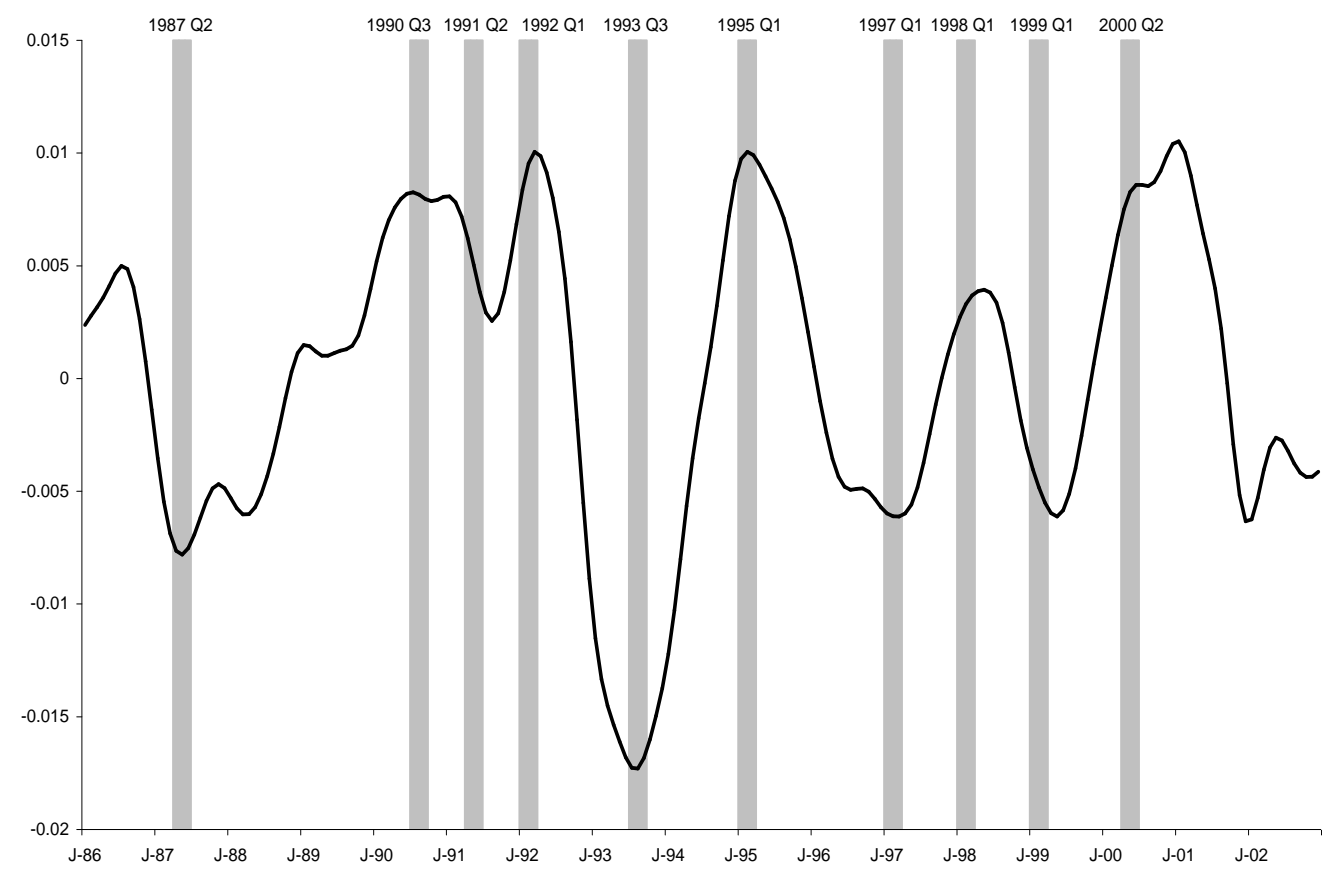

Figure 3: Coincident indicator for the Euro area business cycle with ECB datings.

\subsection{A coincident indicator for growth}

Up to now, the focus has been on the business cycle and how one can exploit the information content of an economy-wide dataset for the assessment of the cyclical position of the economy using a model-based approach. Nevertheless, the policymaker can also be interested in track- 
Table 2: Descriptive statistics for the business cycle and growth indicators

\begin{tabular}{lcccc}
\hline Indicator & mean & stand.dev & minimum & maximum \\
\hline \hline Business cycle & 0.000086 & 0.0066 & -0.017 & 0.01052 \\
Growth & 0.0051 & 0.0038 & -0.0072 & 0.01156 \\
\hline
\end{tabular}

ing economic activity growth. As a by-product, one can obtain a growth indicator with the suggested model-based approach. This can be done by restoring the business cycle indicator with the corresponding estimated trend component and then computing the growth rate. The three-month change of the trend restored business cycle indicator is plotted in figure 4 . The resulting growth indicator can be interpreted as the latent monthly measure of GDP quarterly growth. Some basic statistics of the growth indicator are reported in table 2. From figure 4, one can see that the growth indicator tracks quite well the GDP quarterly growth. Moreover, it is available on a monthly basis and avoids the erratic behaviour of GDP quarterly growth rate which complicates the assessment of the current economic situation. By disregarding the irregular component one allows to obtain a smooth growth indicator. In a similar fashion as for the business cycle indicator, one can also use the $h$-step ahead forecast of the coincident growth indicator as the leading one.

In figure 5, we plot our growth indicator together with EuroCOIN. The EuroCOIN indicator was suggested by Altissimo, Bassanetti, Cristadoro, Forni, Lippi, Reichlin, and Veronese (2001) and it is also intended to be a monthly coincident indicator for the Euro area economic growth. It is based on a generalised dynamic factor model, see Forni, Hallin, Lippi, and Reichlin (2000), and resorts to a huge dataset (almost a thousand series referring to the six major Euro area countries). The model underlying EuroCOIN can be described briefly in the following way. Each series in the dataset is modelled as the sum of two uncorrelated components, the common and the idiosyncratic. The common component is driven by a small number of factors common to all variables but possibly loaded with different lag structures, while the idiosyncratic component is specific to each variable. EuroCOIN is the common component of the GDP growth rate ${ }^{4}$ after discarding its high frequency component. Although this approach allows for more general cyclical dynamics when compared with our model, it also presents additional drawbacks. For instance, it requires the detrending of the series prior to dynamic factor analysis and one cannot use simultaneously series of different periodicity. Moreover, with the estimation of only two parameters for each series, the common cycle factor load and the shift, we were able to get a quite similar outcome.

\footnotetext{
${ }^{4}$ As mentioned earlier, the GDP monthly series used as input was obtained through the linear interpolation of the quarterly figures.
} 


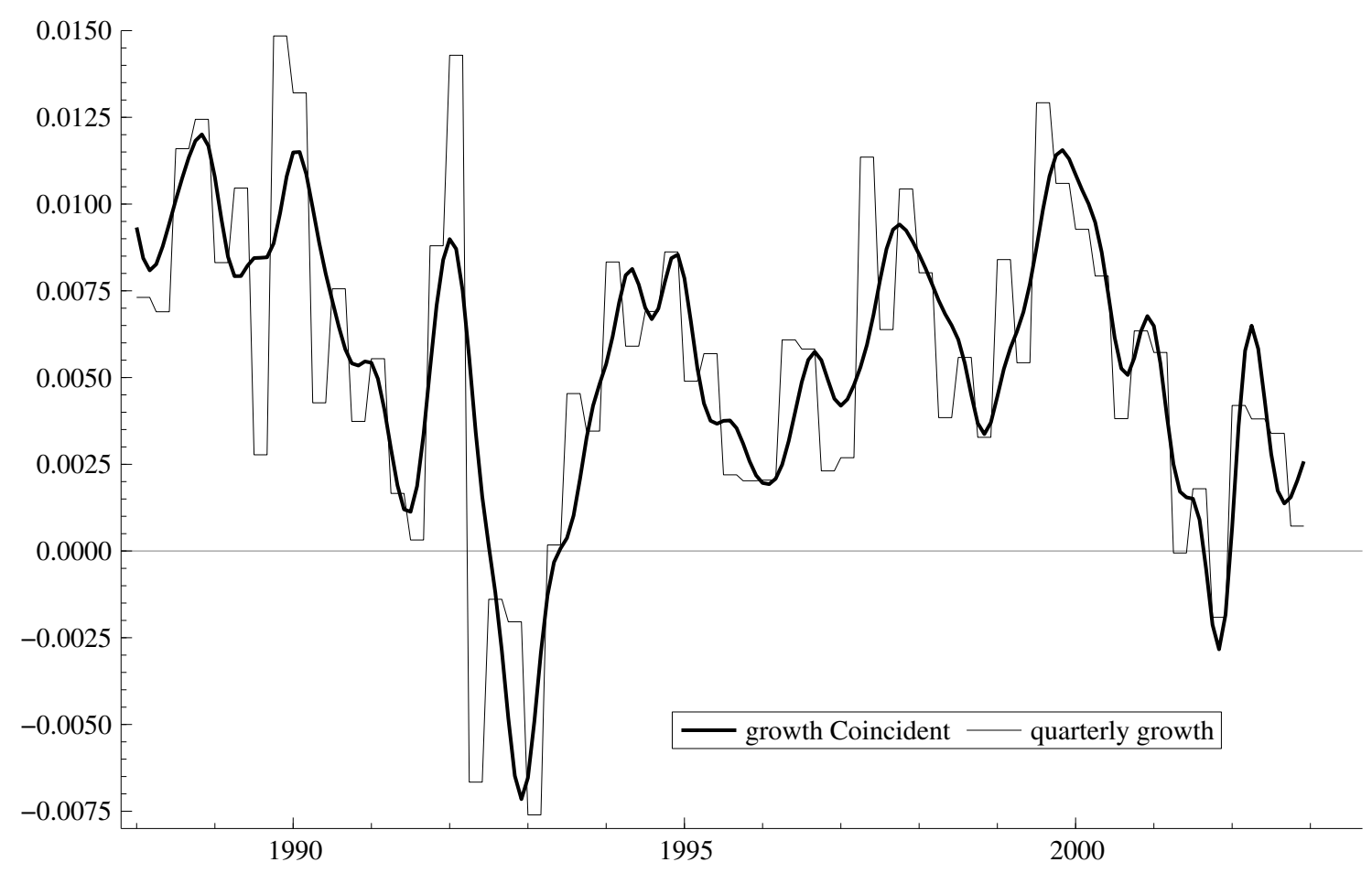

Figure 4: Euro area quarterly GDP growth and its model-based monthly coincident indicator.

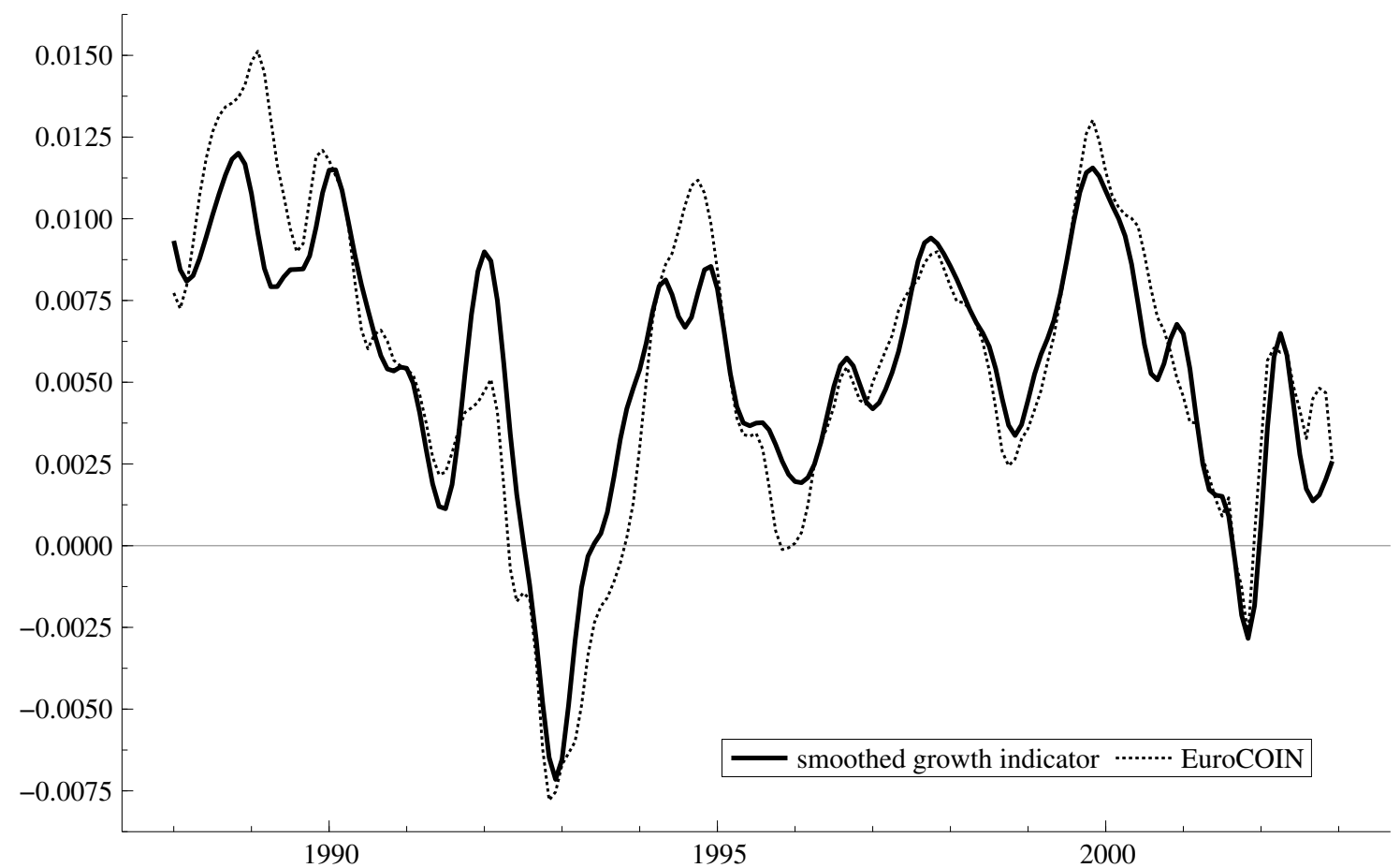

Figure 5: EuroCOIN and our model-based coincident indicator for growth. 


\subsection{Revisions}

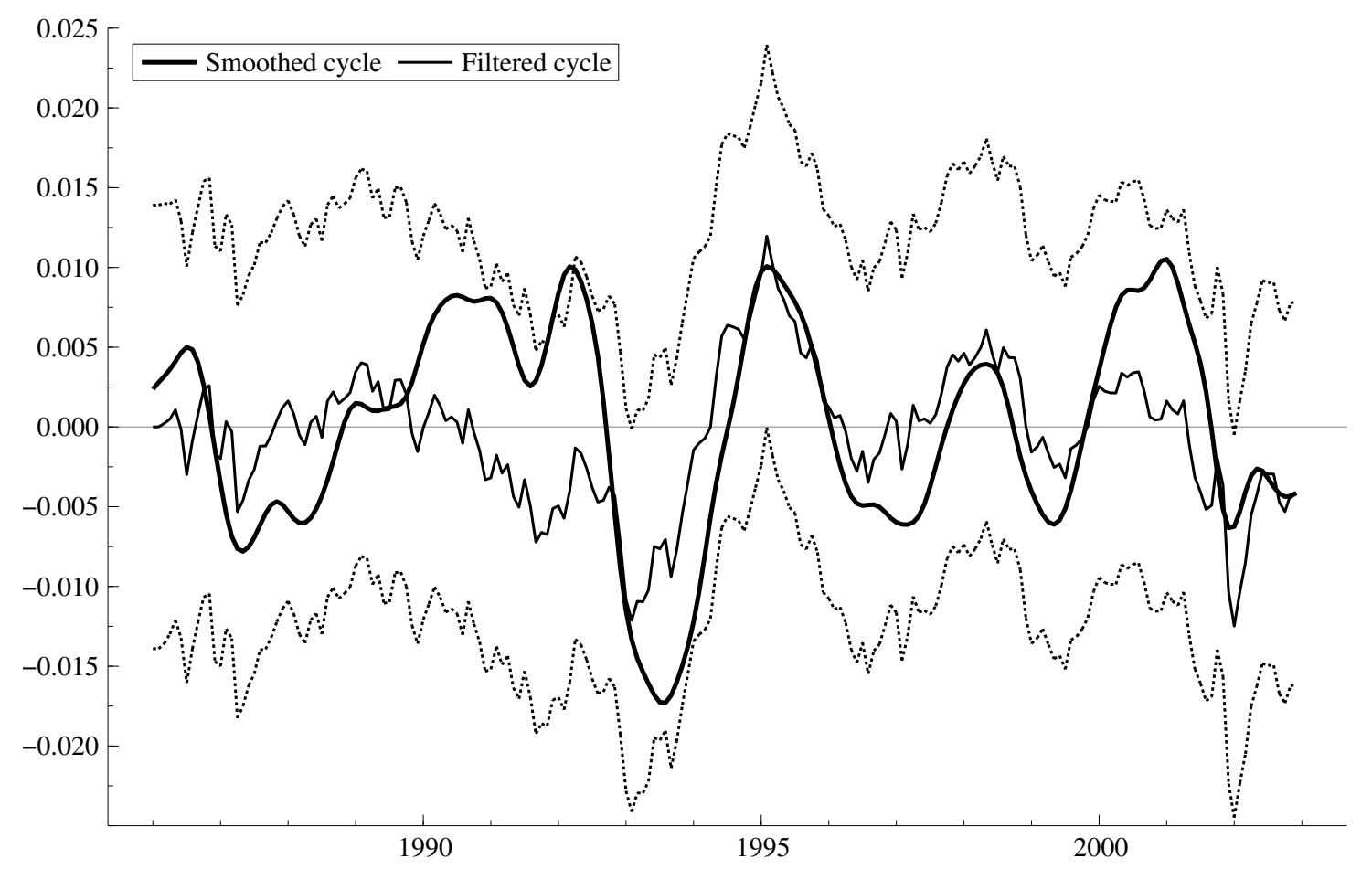

Figure 6: Smoothed and filtered estimates of the business cycle coincident indicator.

A relevant issue regarding both business cycle and growth indicators refers to their real-time reliability. In practice, the indicators are subject to revisions over time due to data revisions and to their re-computation when new data becomes available. Although it is not possible to evaluate the consequences of the first potential source of revisions, due to the lack of data vintages, one can assess the second one by comparing the smoothed and the filtered versions of the indicator. Since the filtered estimate is the one conditional on the information available at each moment whereas the smoothed estimate is the one based on the full set of data, one can compare them to evaluate the magnitude of the revisions. In figure 6 we plot both the filtered and the smoothed versions of the business cycle coincident indicator. One can see that the smoothed falls, in general, within the $95 \%$ confidence interval for the filtered and that the resemblance is more pronounced in the second half of the sample. Additionally, we also compute several measures to assess the importance of the revisions, namely, the cross-correlation between the filtered and smoothed estimates of the indicator, the noise-to-signal ratio (i.e. the ratio of the standard deviation of the revisions to the standard deviation of the smoothed estimate) and the sign concordance (i.e. the proportion of time that smoothed and filtered estimates share the same sign). The results are presented in table 3. The results' analysis should take into account that it is extremely hard to estimate the output gap in a real-time scenario since only with the advance of time can one be increasingly more accurate about the cyclical position at a 
given period. For example, Orphanides and van Norden (2002) have shown that the reliability of real-time estimates of the output gap, whatever the method used to obtain it, is quite low. Nevertheless, the filtered estimate presents a reasonable correlation with the smoothed one, in particular, in the second half of the sample (0.75), as well as a relatively high sign concordance $(0.84)$.

Table 3: Revision and reliability statistics for the business cycle coincident indicator

\begin{tabular}{lccccccc}
\hline Period & mean & stand.dev & min & max & $\begin{array}{c}\text { ratio } \\
\text { stand.dev }\end{array}$ & $\begin{array}{c}\text { corr. } \\
\text { estimates }\end{array}$ & $\begin{array}{c}\text { sign } \\
\text { conc. }\end{array}$ \\
\hline \hline $1989-2002$ & -0.0011 & 0.0059 & -0.015 & 0.0108 & 0.84 & 0.55 & 0.72 \\
$1993-2002$ & 0.00099 & 0.0048 & -0.0099 & 0.0108 & 0.66 & 0.75 & 0.84 \\
\hline
\end{tabular}

The ratio stand.dev is the ratio of the standard deviation of the revisions against the standard deviation of the final estimates. The correlation estimates (corr. estimates) is the contemporaneous correlation between the real time (filtered) estimates and the final (smoothed) estimates of the business cycle. The sign concordance (sign conc.) is the percentage of times that the sign of the real time and final estimates are equal.

The same evaluation can be done for the growth indicator (see figure 7 and table 4). Interestingly, the growth indicator seems to be less affected by revisions. It presents a higher correlation (0.84) and a lower noise-to-signal ratio than its business cycle counterpart. Unfortunately, one cannot compare the revisions of our growth indicator with the ones of EuroCOIN since Altissimo, Bassanetti, Cristadoro, Forni, Lippi, Reichlin, and Veronese (2001) did not perform this evaluation.

Although the indicators are inevitably affected by revisions the results as a whole seem quite promising.

Table 4: Revision and reliability statistics for the growth indicator

\begin{tabular}{lcccccc}
\hline Period & mean & stand.dev & $\min$ & $\max$ & $\begin{array}{c}\text { ratio } \\
\text { stand.dev }\end{array}$ & $\begin{array}{c}\text { corr. } \\
\text { estimates }\end{array}$ \\
\hline \hline $1989-2002$ & -0.000046 & 0.0024 & -0.0086 & 0.0073 & 0.64 & 0.84 \\
$1993-2002$ & -0.000024 & 0.0020 & -0.0056 & 0.0055 & 0.59 & 0.84 \\
\hline
\end{tabular}

The ratio stand.dev is the ratio of the standard deviation of the revisions against the standard deviation of the final estimates. The correlation estimates (corr. estimates) is the contemporaneous correlation between the real time estimates and the final estimates of the growth indicator. 


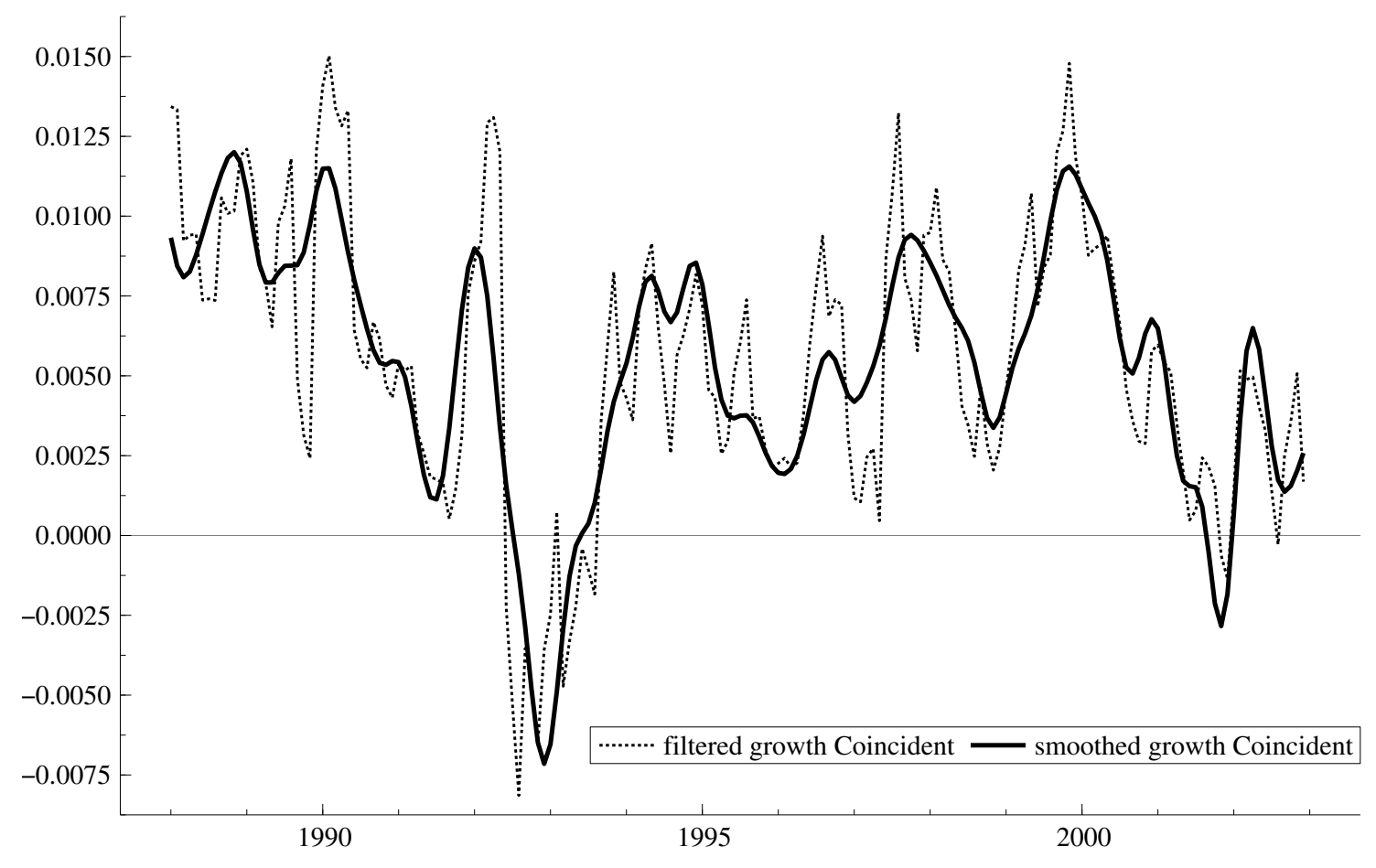

Figure 7: Filtered and smoothed estimates of the coincident indicator for growth.

\section{Conclusion}

In this paper we propose a new model-based approach for obtaining growth and business cycle indicators simultaneously. A multivariate unobserved components time series model is adopted that incorporates two recent and relevant contributions: (i) the inclusion of a cycle component with properties similar to those of band-pass filtered series, developed by Harvey and Trimbur (2003); (ii) the incorporation of phase shifts within the cycle component, proposed by Rünstler (2002). In particular, we consider the cycle component with band-pass filter properties by having it as a common cycle component that allows for phase shifts. The resulting multivariate model is used to extract trend and cycle components using several economic time series for the Euro area. The innovations to the cycle component of each time series are common but their contribution is derived from a filter that depends only on three parameters. One is the frequency of the cycle, which we fix so as to assure that the typical business cycle frequencies are captured. Other accounts for phase shifts and the other accounts for the relative contribution of the cycle to the dynamics of the individual time series. The base cycle is associated with real GDP and it resumes to our business cycle indicator. By discarding the irregular component of GDP, a smooth growth indicator can also be constructed. Due to the modelling of phase shifts, this approach allows to exploit information from multiple economic series, regardless their coincident, leading or lagging nature, to obtain estimates of the current cyclical position 
and of the growth state of the economy. Moreover, despite the fact that GDP is only available quarterly and the remaining indicators are monthly, we are able to incorporate information from GDP without the ad-hoc construction of an artificial monthly GDP series. The use of quarterly GDP together with monthly indicators is straightforward within the state space framework. The resulting indicators are based on nine key economic time series and appear to be in line with common wisdom regarding Euro area economic developments. In particular, our growth indicator is very close to EuroCOIN, which is obtained using hundreds of series as input and is based on a more involved approach. The revisions' evaluation of our business cycle indicator confirms the difficulty of assessing the cyclical position in real-time, as reported by Orphanides and van Norden (2002). Nevertheless, the real-time reliability of the growth indicator is shown to be substantially higher.

\section{Appendix A: State space representation of model}

A state space representation consists of two equations, the observation equation for $y_{t}$ and the transition equation that describes the dynamic process through the state vector. The model (6) requires the following state vector,

$$
\alpha_{t}=\left(\begin{array}{c}
\alpha_{t}^{\mu} \\
\alpha_{t}^{\psi}
\end{array}\right), \quad \alpha_{t}^{\mu}=\left(\begin{array}{l}
\mu_{t}^{(m)} \\
\mu_{t}^{(m-1)} \\
\vdots \\
\mu_{t}^{(1)}
\end{array}\right), \quad \alpha_{t}^{\psi}=\left(\begin{array}{l}
\psi_{t}^{(k)} \\
\psi_{t}^{(k)+} \\
\psi_{t}^{(k-1)} \\
\psi_{t}^{(k-1)+} \\
\vdots \\
\psi_{t}^{(1)+}
\end{array}\right),
$$

with $\mu_{t}^{(j)}=\left(\mu_{1 t}^{(j)}, \ldots, \mu_{N t}^{(j)}\right)^{\prime}$ and $\mu_{i t}^{(j)}$ as in $(9)$ for $i=1, \ldots, N, j=1, \ldots, m$ and $t=1, \ldots, n$. The observation equation is a linear combination of the state vector together with observation noise, that is

$$
y_{i t}=\left\{\begin{array}{lllll}
e_{i} & 0_{1 \times(m-1) N} & \delta_{i} \cos \left(\lambda \xi_{i}\right) & \delta_{i} \sin \left(\lambda \xi_{i}\right) & 0_{1 \times 2(k-1)}
\end{array}\right\} \alpha_{t}+\varepsilon_{i t}, \quad i=1, \ldots, N,
$$

where $e_{i}$ is the $i$-th row of the $N \times N$ identity matrix $I_{N}$ and $0_{p \times q}$ is the $p \times q$ matrix of zeros.

Define $A_{p}$ as a $p \times p$ matrix with zero elements except for elements $(i, i+1)$ that are equal to unity for $i=1, \ldots, p-1$. The transition equation for the state vector is then given by

$$
\alpha_{t+1}=\left(\begin{array}{c}
\alpha_{t+1}^{\mu} \\
\alpha_{t+1}^{\psi}
\end{array}\right)=\left(\operatorname{diag}\left[I_{m} \otimes I_{N} \quad I_{k} \otimes T_{\lambda}\right]+\operatorname{diag}\left[A_{m} \otimes I_{N} \quad A_{k} \otimes I_{2}\right]\right) \alpha_{t}+\eta_{t}
$$

where $\eta_{t}^{\prime}=\left(0_{1 \times(m-1) N}, \zeta_{1 t} \ldots \zeta_{N, t}, 0_{1 \times 2(k-1)}, \kappa_{t}, \kappa_{t}^{+}\right)$,

$$
T_{\lambda}=\left[\begin{array}{cc}
\cos \lambda & \sin \lambda \\
-\sin \lambda & \cos \lambda
\end{array}\right], \quad \operatorname{Var}\left(\eta_{t}\right)=\operatorname{diag}\left[\begin{array}{cccc}
0_{(m-1) N \times(m-1) N} & \Sigma_{\zeta} & 0_{2(k-1) \times 2(k-1)} & \sigma_{\kappa}^{2} I_{2}
\end{array}\right],
$$


and $\Sigma_{\zeta}$ is a diagonal $N \times N$ matrix with $\sigma_{i, \zeta}^{2}$ of (8) as its $i$-th diagonal element. We note that diag [..$]$ refers to a block diagonal matrix. The initial state vector $\alpha_{1}^{\mu}$ will be treated as a diffuse vector and appropriate amendments to the Kalman filter and related algorithms need to be made, see Durbin and Koopman (2001, Chapter 4) for a discussion. The stochastic properties of the initial state vector $\alpha_{1}^{\psi}$ are implied by mean zero and variance matrix $P$ which is the solution of

$$
P=\left[I_{k} \otimes T_{\lambda}+A_{k} \otimes I_{2}\right] P\left[I_{k} \otimes T_{\lambda}+A_{k} \otimes I_{2}\right]^{\prime}+\operatorname{diag}\left[\begin{array}{ll}
0_{2(k-1) \times 2(k-1)} & \left.\sigma_{\kappa}^{2} I_{2}\right]
\end{array}\right.
$$

We note that the solution of $A=B A B^{\prime}+C$ is $\operatorname{vec}(A)=(I-B \otimes B)^{-1} \operatorname{vec}(C)$, see Magnus and Neudecker (1988, Theorem 2, p.30). Explicit formulations for $P$ are reported in Trimbur (2002).

\section{Appendix B: Data description}

As far as possible, we used series from Euro area official statistical sources, i.e., Eurostat, the European Commission and the European Central Bank. However, the available data regarding the Euro area as a whole is rather limited, in particular, in terms of time span. Therefore, in some cases, we had to chain the official time series with an Euro area aggregate proxy, obtained by weighting country level data with 1995 (PPP) GDP weights, which were rescaled whenever there was any missing data for a country. The sample runs from the beginning of 1986 up to the end of 2002. Regarding real GDP, the seasonally adjusted series provided by the Eurostat only starts in the first quarter of 1991. Therefore, from 1991 backwards we used the series provided by Fagan, Henry, and Mestre (2001) to obtain a quarterly series for the above mentioned sample period. All confidence indicators are seasonally adjusted and provided by the European Commission. The industrial production volume index is seasonally adjusted and provided by the Eurostat. The unemployment series is also seasonally adjusted and provided by the Eurostat. Since there is no official series before June 1991, an aggregate series was obtained, in this particular case, by summing country level data. The retail sales volume index is seasonally adjusted and provided by the Eurostat since 1996. To obtain a longer time span series, we resorted to country level data provided by the OECD. Regarding nominal interest rates, since both short and long-term interest rates are made available by the ECB only for the period after January 1994, we had also to use OECD country level data. The nominal shortterm interest rate refers to the 3-month deposit money market rate and the nonimal long-term interest rate refers to the 10-year government bond yield. The yield curve spread is given by the difference between the long and the short-term interest rates. Excluding confidence indicators and interest rate spread, all data are in logarithms. 


\section{References}

Altissimo, F., A. Bassanetti, R. Cristadoro, M. Forni, M. Lippi, L. Reichlin, and G. Veronese (2001). EuroCOIN: a real time coincident indicator of the euro area business cycle. Working paper no. 3108, CEPR, London.

Baxter, M. and R. King (1999). Measuring business cycles: approximate band-pass filters for economic time series. Rev. Economics and Statistics 81, 575-93.

Christiano, L. and T. Fitzgerald (2003). The Band-Pass filter. International Economic Review $44,435-65$.

Doornik, J. A. (2001). Object-Oriented Matrix Programming using Ox 3.0 (4th ed.). London: Timberlake Consultants Ltd. See http://www.nuff.ox.ac.uk/Users/Doornik.

Durbin, J. and S. J. Koopman (2001). Time Series Analysis by State Space Methods. Oxford: Oxford University Press.

ECB (2001). The information content of composite indicators of the Euro area business cycle. Monthly bulletin, November, 39-50, European Central Bank (ECB), Frankfurt.

ECB (2002). Characteristics of the Euro area business cycle in the 1990s. Monthly bulletin, July, 39-49, European Central Bank (ECB), Frankfurt.

Fagan, G., J. Henry, and R. Mestre (2001). An area wide model for the Euro area. Working paper no. 42, European Central Bank (ECB), Frankfurt.

Forni, M., M. Hallin, M. Lippi, and L. Reichlin (2000). The generalized factor model: identification and estimation. Rev. Economics and Statistics 82, 540-54.

Gomez, V. (2001). The use of Butterworth filters for trend and cycle estimation in economic time series. J. Business and Economic Statist. 19, 365-73.

Harvey, A. C. (1989). Forecasting, Structural Time Series Models and the Kalman Filter. Cambridge: Cambridge University Press.

Harvey, A. C. and A. Jaeger (1993). Detrending, stylised facts and the business cycle. J. Applied Econometrics 8, 231-47.

Harvey, A. C. and S. J. Koopman (1997). Multivariate structural time series models. In C. Heij, H. Schumacher, B. Hanzon, and C. Praagman (Eds.), Systematic dynamics in economic and financial models, pp. 269-98. Chichester: John Wiley and Sons.

Harvey, A. C. and T. Trimbur (2003). Generalised model-based filters for extracting trends and cycles in economic time series. Rev. Economics and Statistics 85, 244-55.

Hodrick, R. J. and E. C. Prescott (1997). Postwar U.S. Business Cycles: An empirical investigation. Journal of Money, Credit and Banking 29, 1-16.

Kitagawa, G. and W. Gersch (1996). Smoothness Priors Analysis of Time Series. New York: Springer Verlag. 
Koopman, S. J. and J. Durbin (2000). Fast filtering and smoothing for multivariate state space models. J. Time Series Analysis 21, 281-96.

Koopman, S. J., N. Shephard, and J. A. Doornik (1999). Statistical algorithms for models in state space form using SsfPack 2.2. Econometrics Journal 2, 113-66. http://www.ssfpack.com/.

Magnus, J. R. and H. Neudecker (1988). Matrix Differential Calculus with Applications in Statistics and Econometrics. New York: Wiley.

Mariano, R. S. and Y. Murasawa (2003). A new coincident index of Business cycles based on monthly and quarterly series. J. Applied Econometrics 18, 427-43.

Orphanides, A. and S. van Norden (2002). The unreliability of output-gap estimates in real time. Rev. Economics and Statistics 84, 569-83.

Rünstler, G. (2002). Modelling phase shifts among stochastic cycles. Mimeo, European Central Bank (ECB), Frankfurt.

Stock, J. and M. W. Watson (1989). New indexes of coincident and leading economic indicators. Macroeconomics annual 1989, NBER, New York.

Stock, J. and M. W. Watson (1999). Business cycle fluctuations in US macroeconomic time series. In J. B. Taylor and M. Woodford (Eds.), Handbook of Macroeconomics, pp. 3-64. Amsterdam: Elsevier Science Publishers.

Trimbur, T. (2002). Properties of a general class of stochastic cycles. Working paper, University of Cambridge.

Young, P. C. (1984). Recursive Estimation and Time Series Analysis. New York: SpringerVerlag. 